\title{
Electronic-Nose Technology and its Application -A Systematic Survey
}

\author{
Pranjal kalita $^{1}$, Manash protim saikia ${ }^{2}$, N.H.Singh ${ }^{3}$ \\ Dept. of Electronics \& Communication Engineering, Dibrugarh University Institute Of Engineering \& Technology, \\ Dibrugarh, Assam ,India ${ }^{1,2,3}$
}

\begin{abstract}
Electronic Nose is one of the most advance device in the field of sensor technology. It is responsible for the automated detection and classification of gases, odors and vapors. This paper reviews the three components of Electronic Nose i.e. sample handling, detection system, data processing system. It outlines the range of sensors their principles, advantages and disadvantages. It describe the data processing through pattern recognition process. It also outlines the enormous benefits of Electronic Nose in the field of food control, clinical diagnosis, cosmetics, environmental factors, garbage control and detection of plant diseases.
\end{abstract}

Keywords: E-Nose, VOC, ANN, ART.

\section{INTRODUCTION}

E-Nose is a measuring instrument that is designed to mimic the mammalian olfactory system. With the advancement in sensor technology, biochemistry, electronics, artificial intelligence it is possible to design the biological olfactory system within an pat instrumentcapable of identifying and classifying the aroma mixture. Its capability of recognition of the mixture of organic sample rather than recognizing individual chemical species in the sample make this device unique from other analytical instruments. Nowadays there are various models of e-nose to separate the complex mixture that contain different type of volatile organic compound (VOC). The electronic nose has a diverse application in the field of food analysis, clinical diagnosis, [2]. environmental control, cosmetic industry and many more.

\section{Basic Design and Operation of E-Nose:}

Any detection device will be considered as electronic nose if and only if the device contain array sensors that can mimic the mammalian olfactory system and can sense the volatile organic compound.

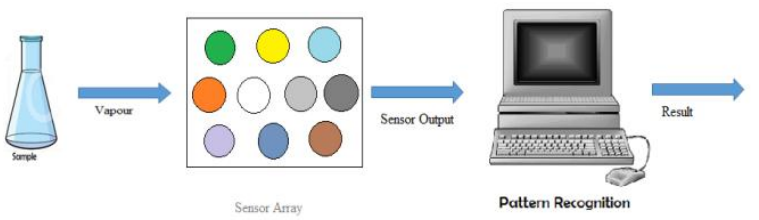

Figure 1: Schematic diagram of an electronic nose An electronic nose consist of sampling handling process, detection process done by multi sensor array, a data processing unit such as artificial neural network, a digital pattern recognition algorithm and a reference library[1].The aroma delivery system transfers the volatile organic molecule from the source to the sensor array system. The multi sensor array composed of different sensors that will respond to wide range of chemicals. The sensors are placed in a closed chamber maintained at constant temperature and humidity. A digital signature (pattern) will be generated by collectively gathering and integrating the outputs of different sensors.Basically transistor will convert the chemical signal to electrical signal, amplifies and condition it. A digital converter is used to convert the electrical signal in analog form to digital form. The simple or complex mixture is characterized by a unique digital aroma signature pattern.A computer will read the digital signal and displays the output.

A pattern recognition algorithm observe the difference between the pattern of all analyte type in the reference library. The process will be continued and the results were added in the reference library to which unknown samples are to be compared. Unknown sample can be identified on the basis of the aroma elements that the analyte pattern has in common with the pattern present in the reference library

\section{Prototype of Electronic-Nose:}

Prototype electronic noses, shown in Figure 2, is composed of an array of nine tin oxide vapor sensors, a humidity sensor, and a temperature sensor coupled with an ANN. Two types of ANNs were constructed for this prototype: the standard multilayer feed-forward network trained with the back propagation algorithm and the fuzzy ART map algorithm. During operation a chemical vapor is blown across the array, the sensor signals are digitized and fed into the computer, and the ANN (implemented in software) then identifies the chemical. This identification time is limited only by the response time of the chemical sensors, which is on the order of seconds. This prototype nose has been used to identify common household chemicals by their odor.

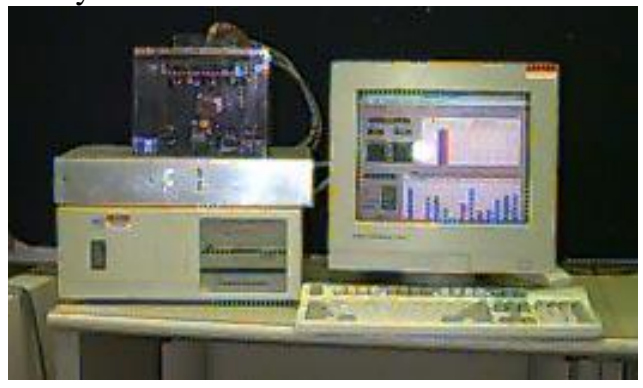

Figure 2: Photograph of the prototype electronic nose 
Figure 3 illustrates the structure of the ANN. The nine tinoxide sensors are commercially available, Taguchi-type gas sensors. (Sensor 1, TGS 109; Sensors 2 and 3, TGS 822; Sensor 4, TGS 813; Sensor 5, TGS 821; Sensor 6, TGS 824; Sensor 7, TGS 825; Sensor 8, TGS 842; and Sensor 9, TGS 880). Exposure of a tin-oxide sensor to a vapor produces a large change in its electrical resistance. The humidity sensor (Sensor 10: NH-02) and the temperature sensor (Sensors 11: 5KD-5) are used to monitor the conditions of the experiment and are also fed into the ANN.

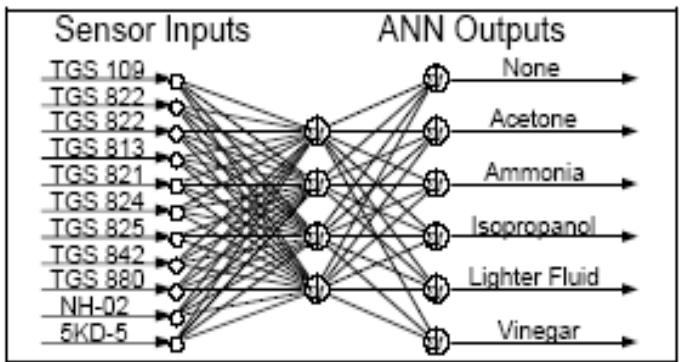

Figure 3: Structure of the back propagation ANN used in the prototype to identify household chemicals

Although each sensor is designed for a specific chemical, each responds to a wide variety of chemicals. Collectively, these sensors respond with unique signatures patterns) to different chemicals. During the training process, various chemicals with known mixtures are presented to the system. By training on samples of various chemicals, the ANN learns to recognize the different chemicals.

This prototype nose has been tested on a variety of household and office supply chemicals including acetone, ammonia, ethanol, glass cleaner, contact cement, correction fluid, lighter fluid, methanol, rubber cement and vinegar. For the results shown in the paper, five of these chemicals were used: acetone, ammonia, lighter fluid, and vinegar. Another category, "none" was used to denote the absence of all chemicals except those normally found in the air which resulted in six output categories from the ANN.

\section{Backpropagation}

Architecture: 11-11-6 feedforward

Activation: Logistic Sigmoidal

Learning Rate: 0.10

Momentum: 0.90

No. of Epochs: 1369

\section{Fuzzy ARTmap}

Training Vigilance: 0.98

Testing Vigilance: 0.80

No. of Epochs: 3

During the training process, various chemicals with known mixtures are presented to the system. By training on samples of various chemicals, the ANN learns to recognize the different chemicals.Both networks were trained using randomly selected sample sensor inputs. Table 1 summarizes one set of network performances for novel sensor inputs.

\begin{tabular}{|l|l|l|l|}
\hline $\begin{array}{l}\text { Num } \\
\text { Train }\end{array}$ & $\begin{array}{l}\text { Num } \\
\text { Test }\end{array}$ & $\begin{array}{l}\text { Input } \\
\text { Substance }\end{array}$ & $\begin{array}{l}\text { \% } \\
\text { correct } \\
\text { BP } \\
\text { FA }\end{array}$ \\
\hline 67 & 28 & None & $\begin{array}{l}96.4 \\
96.4\end{array}$ \\
\hline 75 & 22 & Acetone & 100 \\
& & Ammonia & 100 \\
\hline 64 & 14 & 100 \\
\hline 93 & 28 & Isopropanol & 92.9 \\
& & Ammonia & 00.0 \\
\hline 5 & 3 & \&Isopr. & 66.7 \\
\hline 106 & 25 & Lighter & 100 \\
& & Fluid & 96.0 \\
\hline 74 & 27 & Amm\&Lig & $\begin{array}{l}100 \\
92.6\end{array}$ \\
\hline 66 & 21 & Fluid & 81.0 \\
& & Vinegar & 95.2 \\
\hline 68 & 26 & Amm\& & 92.3 \\
& & Vinegar & 76.9 \\
\hline 1 & 2 & Isopr\& & 00.0 \\
Vinegar & 00.0 \\
\hline
\end{tabular}

Table 1. ANN performance for backpropagation (BP) andfuzzy ARTmap (FA)

\section{Description of Electronic Nose:}

Electronic nose composed of three elements: sample handling, detection system and data processing. Each of them will be discussed in brief.

\section{Sample handling system}

The quality of the analysis can be greatly improved by adopting various sampling methods.To introduce the volatile compound into the detection system of the electronic noes various sampling techniques are performed:

\section{Static Headspace Extraction (SHS):}

Volatile components can be analysed quantitatively and qualitatively using this technique. This process is based on placing the sample hermetically in a sealed vial and extracting the volatile components once the equilibrium is established between the matrix and the gaseous phase. Sample temperature, equilibration time, vial size, sample quantity are some parameters that have to be optimized for efficiency,sensitivity, quantitation and reproductibility[3, $4,5]$.

\section{Dynamic Headspace Extraction (DHS):}

Dynamic Headspace Extraction(DHS) or Purge and Trap is used in high sensitivity application. It is based on the volatility of the analytes to achieve extraction from the matrix. Here in this method the volatile components are purged by a stream of inert gas and trapped onto an absorbent. The trapped molecule are desorbed by heating and is introduced into the detection system which will increase the sensitivity for analysis $[3,5,6]$. 
3. Solid-Phase-Microextraction(SPME):

Solid-phase-microextraction use uses a silica fiber coated with an absorbent in the headspace of the sample. The volatile component is extracted from the sample onto the fiber. The absorbed compounds are desorbed by heating and then introduced into the detection system. Parameters like nature of absorbent deposited on fiber, equilibration time, sample temperature and extraction duration are to be optimized $[3,5]$.

\section{Detection system:}

The multisensory array is considered as the most important component of electronic nose as it carry out an identical role to the human olfactory nerve.

An ideal sensor must have these following characteristics to fulfill various criteria:Sensors should have high sensitivity toward group of chemical compounds for detection, Sensors should have relatively low selectivity so that it could be sensitive toward a wide range of chemicals, For uncontrolled environmental conditions application, it should have low sensitivity to variable parameters such as temperature and humidity, Sensors should have short calibration and training requirements, fast recovery time between runs and maintenance procedure, Sensors should operate at low cost, Sensors should have short recording as well as analysis time, A sensor should respond to various compounds in the headspace, It should have high stability, high reproducibility and reliability, It should process the data output easily [7, 8, 9].

An electronic nose consist of array of gas sensors for the detection purpose. An electrochemical gas sensor is a device that converts chemical quantity into an electrical signal. Its operation is based on the interaction of gaseous molecule with sensor-coating materials which regulates the electrical current passing through the sensors. Those electrical signals were detected by a transducer that converts the modulation into a recordable electronic signal. There are various types of electrochemical sensors such as: Metal-oxide semiconductor sensors, metal oxide semiconductor field effect transistor, conducting polymer gas sensors,Piezoelectricsensors,optical sensors and many different types of sensors.

\section{Metal -oxide semiconductor (MOS) sensors:}

Metal-Oxide semiconductor sensors are widely used class of gas sensors as they include wide range of electronic, chemical, optical properties .The basic principle operation is the interaction between the chemically absorbed oxygen species on the MOS surface and gaseous molecule on the metal oxide surface leads to change in conductance [10]. The metal oxide coating either consist of n-type (zinc oxide, tin dioxide, titanium dioxide) or p-type material (nickel oxide, cobalt oxide). The n-type material will respond to oxidizing compounds whereas p-type material will respond to reducing compounds. Thermal excitation of n-type materials leads to excess of electron in its conduction band which tends to increase the reactivity of oxidizing molecule. Whereas thermal excitation of p-type material will results in the electron deficiency in the valance band and that will increase the reactivity of reducing compound [11].

The sensitivity of a metal oxide sensor is given as $\Delta R / R_{b}$

$c(g a s)$

Where $\Delta R=R-R_{b}$ for oxidizing gases and $\Delta R=R_{b}-R$ for reducing gases.

Here $R_{b}$ is the baseline resistance and $R$ is the resistance on exposure to the odour and $c$ ( $g a s)$ is the concentration of gas $[12,13]$.

MOS sensors operates in very high temperature (300$500^{\circ} \mathrm{C}$ ). At this high temperature the volatile organic compound which is transferred to the sensor will get combusted totally to carbon dioxide and water on the surface of the metal oxide, that leads to change in the resistance. The reaction mechanism is based on exchange of oxygen between the VOC and the metal oxide film [7]. MOS sensors are selective toward various chemicals by doping the film with a noble catalytic metal (palladium or platinum) or it can be made more selective by changing the working temperature within $50-400^{\circ} \mathrm{C}$ [7].

\section{Metal-oxide semiconductor field effect} transistor sensor:

The MOSFET is a transducer device used to convert chemical changes into electrical signal. A MOSFET sensor is a combination of three layers: silicon semiconductor, silicon oxide insulator and catalytic metal which act as gate terminal of the MOSFET[13]. Normally the transistor operation is based on three contacts, flow of current from drain to source and the gate will act as the control to regulate the current through the transistor. The voltage is applied across the gate and drain terminal which will generate electric field which results in the conductivity of the transistor. When a polar compound and the gate metal interacts, it generates electric field and respond is recorded by the transistor $[13,14]$. The gate can be either thick (100-200 nm)or thin (6-20 nm). When the gate is thick the sensor will respond to all molecule that dissociate hydrogen in the catalytic metal surface and the sensor will remain less sensitive toward non hydrogen releasing molecules. But in case of thin gate structure the sensor will respond to non hydrogen releasing molecule as well [15].

\section{Conducting polymer gas sensor:}

This sensor is the most widely used sensor element in electronic noes because of their reversible physic-chemical properties. It consist of substrate (silicon), pair of goldplated electrons, conducting polymer coating as sensing element [7]. When voltage is passed across the electrode, a current flows through the conducting polymer. The interaction between the polymer and the volatile compound alters the electron flow in the system which results in change in the resistance [16]. 
This sensor possess various properties: it is highly sensitive, has short response time, can be easily synthesized, have good mechanical properties, can be operated at ambient temperature condition.

\section{Piezoelectric sensors:}

Piezoelectric sensors are based on the piezoelectric effect which states that electric potential generated by a crystal is proportional to applied mechanical stress. Piezoelectric crystal have a resonant frequency which is proportional to the mass of the crystal in other words it can be concluded as change in the mass of the crystal will result in change in resonance frequency [17].

Two types of piezoelectric sensors are used namely: surface acoustic wave (SAW) and quartz crystal microbalance (QCM). Both sensors is based on the principle that as the mass of piezoelectric sensor coating changes due to absorption of gas, the resonant frequency will change [18].

\subsection{Surface Acoustic Wave Sensor:}

SAW sensor consist of piezoelectric substrate with a transmitting and receiving transducer deposited on the top of the substrate. A sensitive membrane is placed between the transducers. When an ac signal is applied the transmitting transducer, an acoustic two dimensional wave is generated and propagated along the surface of the crystal at a depth of one wavelength at operating frequencies $(100-400 \mathrm{MHz})$. The interaction of analyte results in change in the mass of the sensitive membrane which in turn alters the frequency of the wave [19]. The change in frequency if given by:

$\Delta f=\Delta f_{p} c_{v} K_{p} / \rho_{p}$

Where $\Delta f_{p}$ is the change in frequency caused by the

membrane, $c_{v}$ is the vapor concentration, $K_{p}$ is the

partition coefficient, $\rho_{p}$ is the density of the polymer membrane $[18,19,20]$.

\subsection{Quartz Crystal Microbalance Sensor:}

In case of QCM, with the applied ac voltage the crystal oscillates at its resonant frequency normally between 10 and $30 \mathrm{MHz}$ results in a three dimensional wave, that propagate through the bulk of the crystal. The absorption of gas by the crystal results in increase in its mass which in turn alters the resonant frequency of the crystal. The change in the frequency will therefore lead to the detection of the vapour[18,21].

\section{Optical sensor:}

Optical sensor is based on the fact that when a light from the source falls from a light source on a volatile organic compound results in signal that can be measured in terms of some properties like absorbance, fluorescence, polarization, refractive index, scattering and reflectance. An optical sensor consist of: light source, suitable optics directing the light to or from sensor, sensing material, photodetector for detecting the light signal comes from the sensor $[5,17]$.
Optical sensor generally divided into two types: intrinsic optical and extrinsic optical. In case of intrinsic optical sensor the change in the optical properties such as absorbance, fluorescence, refractive index on the sensing surface can detect the presence of gaseous compound. In extrinsic optical sensoran indicating species is employed to detect the analyte by being attached on an optical substrate.

So it is concluded that based on the unique advantages and disadvantages of an individual sensor types the range of capabilities and its application can be determined. Some factors such as operational expenses, maintenance costs, training cost and ease to operate should be kept in mind while selection of sensors.

\section{Data Processing System}

The data generated by each sensors can be processed using the pattern recognition technique. Recognition of the sample can take place after the sensor output is stored in the computer in a digitized aroma pattern.

The pattern recognition process takes place in four stages: signal processing, dimensionality reduction, prediction and validation [22]. Signal processing is the first step that focus in compensating the sensor drift, extracting the descriptive parameters from the sensor array response and prepares the feature vector for further processing. In dimensionality reduction the initial feature vector dimension is reduced to avoid problems related with highdimensionality datasets. Data classification, regression, clustering is done in the prediction stage. Identification of the unknown odor can be done by classification based on database of prior analysis of known odorants. Regression defines the properties of the analytes. Clustering is used in finding the spatial relationship among the data sample. The estimation true error rate for trained model and parameter setting and model selection can be done by validation.

Pattern recognition is divided into two categories: supervised and unsupervised. In case of supervised pattern recognition method based on the prior analysis of known odor in the database, an unknown odor can be tested and classified. In case of unsupervised pattern recognition method identification of the unknown odor can be achieved by without corresponding odor class description [23]

\section{1. $\quad$ Principal Component Analysis (PCA)}

Principal component analysis (PCA) is an unsupervised pattern recognition technique. It is a linear feature extraction technique.It is used for visualizing the response of an electronic nose in a reduced dimensional space with minimum loss of information. Projecting the data onto fewer dimensions, original data can be condensed so that maximum amount of information is retained in a smaller dimension. It offer faster classification and identification of unknown sample by reducing the detection time. PCA is a linear technique and if the sensors output is not linear then interpolation of features may occur. 


\section{2. $\quad$ Artificial Neural Network (ANN)}

Artificial Neural Network (ANN) is a commonly used technique of supervised pattern recognition. It is an information processing paradigm that mimics the biological nervous system. It consist of large number of interconnected processing elements work parallel to solve a specific task [24]. Its capability to extract information from imprecise data make it useful for pattern recognition. ANN has various advantages: it has the ability to learn to solve the specific task based on the given data (adaptive learning),it is self organized and has real time operation [25].

\subsection{Architecture of Neural Network}

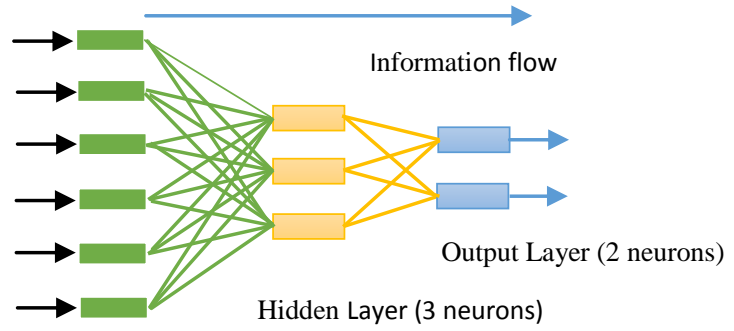

Input Layer (6 neurons)

Figure 4. Back propagation artificial neural for pattern recognition system

The above given artificial neural network composed of three layers: input unit, hidden layer and output layer. The six neuron at the input layer represents the six number of sensor are used in the sensor array. It provides the raw III information to the network. In between the input and output layer there is a hidden layer whose performance is determined by the activities of input unit and the weights on the connection between the input and output unit. And the behavior of output unit is determined by the activity of the hidden unit and the weights between the output and hidden units.

This simple type of network is interesting because the hidden units are free to construct their own representations of the input. The weights between the input and hidden IV. units determine when each hidden unit is active, and so by modifying these weights, a hidden unit can choose what it represents.

\section{Application of Electronic Nose:}

Electronic nose is widely used in different industries, which includes automobile, food, packaging, cosmetic, drug, analytical chemistry and biomedical. The application of e nose include quality control of raw and manufactured products, process design, freshness and maturity (ripeness)monitoring, shelf-life investigations, authenticity assessments of premium products, classification of scents and perfumes, microbial pathogen detection and environmental assessment studies.

The various application of e nose in different industries is discussed below in brief:

I. In food industries:_To maintain and improve food quality now a day's electronic nose is widely used in different food industries for different purposes. Electronic nose is used to determine the flavor or smell along a food production process. In food production where microbiological processes are involved e-nose is applied to bioprocess monitoring, in the production of fermented dairy products. $[27,28]$. It is also used in the manufacturing process of winemaking and black tea. $[27,28]$. During the period of harvesting until consumption e-nose is used to control the ripening process of fruits and other vegetables. The period of harvesting until consumption is called shelf life period. [27,30-38]In food industry one of the most important qualities of food which should be maintained is freshness. E-nose is used in food industries to predict the freshness or spoilage of different food raw material and product. It mostly deals with fish and fish products. [27,39]

II. In medicine: Electronic nose is used as a diagnostic tool in the field of medicine. It is used to identify possible problems by examining odours from the body.[40] For example: gastrointestinal problems, sinus problems, infections, diabetes, and liver problems can be detected by odours in the breath. Odors coming from body fluids can indicate liver and bladder problems.[40,41] Now a days wound infections can be detected with the help of e-nose. With very modern technology application e-nose has been proposed for telesurgery. [40,42]

In environmental monitoring: To perform environmental monitoring and waste management in cost effective manner a technology is explored by Pacific Northwest National Laboratory. This technology includes the development of portable, inexpensive systems capable of real time identification of contaminants in the field.[40] The technology which is used in electronic nose covers all the requirements which are required for environmental monitoring. [40,43]

In garbage control: In today's world garbage is a major cause of pollution. If we consider the scenario of India, Indian cities alone generate more than 100 million tons of solid waste a year. Street corners are piled with trash. Public places and sidewalks are despoiled with filth and litter, rivers and canals act as garbage dumps. Electronic nose technology can be used to minimize this, which can control the amount of garbage gathered in a dustbin or any other places by sensing it and can monitor the environment.

V. In detection of plant disease: To detect the element of plant disease such as fungi and bacteria electronic nose technology can be used. E-Nose can identify PenicilliumAspergillusand Fusarium. These fungi produce mycotoxins during plant growth and cause harm. It also reduced the nutritional quality. So enose is used to detect this plant disease [44]. 


\section{CONCLUSION}

In this paper we discussed about the electronic nose, its ability of identifying and classifying any sample type with high efficiency. Electronic Nose with diverse sensor array make the device highly responsive toward a wide range of analytes.It has various of advantages like it has good sensitivity, can produce rapid results, less expensive as compared to other analytical instruments, easier to operate. But at the same time it has some disadvantages too like negative effect toward temperature and humidity, problem with reproductibility, interference with other gases.

Different types of sensor are reviewed in this paper. The operational principle, advantages and disadvantages of each sensor type is clearly outlined. The Artificial Neural Network used for pattern recognition is designed in such a manner that it mimics the biological nervous system of human being. Thus an electronic sensing refers to the capability of mimicking the mammalian olfactory system using sensor array and pattern recognition system.

\section{REFERENCES}

[1] Gardner, J.W.; Bartlett, P.N. A brief history of electronic noses. Sens. Actuat. B: Chem, 18,1984, pp.211-220

[2] AlphusD.Wilson, Manuela Baietto, Application and Advances in Electronic-Nose Tecnologies, Sensors 9(2009), pp.5099-5148

[3] Miguel Peris, Laura Escuder-Gilabert, A $21^{\text {st }}$ century technique for food control : Electronic noses, AnalyticaChimicaActa, 638(2009), pp.1-15

[4] M. Bernabei, G. Pennazza, M. Santonico, C. Corsi, C. Roscioni, R. Paolesse, C. D. Natale and A. D'Amico, "A Preliminary Study on the Possibility to Diagnose Urinary Tract Cancers by an Electronic Nose," Sensors \&Actua-tors: B. Chemical, Vol. 131, No. 1, 2008, pp. 1-4.

[5] Simeng Chen, Yuchao Wang, Seokheum Choi, Application and Technology of Electronic Nose for Clinical Diagnosis, Open Journal of Applied Biosensor, 2(2013),pp.39-50

[6] S. Kanoh, H. Kobayashi, and K. Motoyoshi, "Exhaled Ethane: An in Vivo Biomarker of Lipid Peroxidation in Interstitial Lung Diseases," Chest, Vol. 128, No. 4, 2005, pp. 2387-2392

[7] E.Schaller, F.Escher, "Electronic Noses and Their Application", Lebensm.-Wiss u-Technol, 31(1998),305-316

[8] Bartlett, P. N., Blair, N. And Gardner, J. W." Electronic nose. Principles,applications and outlook". ASIC, 15e Colloque, Montpellier, 1993, pp. 478-486.

[9] DEMARNE, V. AND SANJINE' S, R. "Thin film semiconducting metal oxide gas sensors". GasSensors, 1992, pp.89-116

[10] J. W. Gardner and P. N. Bartlett, "Electronic Noses: Prin- ciples and Applications," Oxford University Press, Ox- ford, 1999

[11] Mielle, P. "Electronic noses: Towards the objective instrumental characterization of food aroma". Trends inFood Science \& Technology, Special Issue on FlavourPerception, 7(1996), pp.432-438

[12] Steffes, H., Imawan, C., Solzbacher, F. and Obermeier, "Enhancement of no2 sensing properties ofin2o3-based thin films using an au or ti surface modification", Sensors and Actuators B: Chemical, Vol. 78, 2001, pp. 106-12.

[13] K.Arshak, E. Moore,G.M.Lyons, J.Harris, S.Clifford, A Review of Gas Sensors Employed in Electronic Nose Applications, Sensor Review, Vol 24(2),2004,181-198.

[14] Eisele, I., Doll, T. and Burgmair, "Low power gas detection with fet sensors", Sensors andActuators B:Chemical, Vol. 78, 2001, pp. 19-25.

[15] Lundstro." M, I., Spetz, A., WINQUIST, F., ACKELID, U. ANDSUNDGREN, H. "Catalytic metals and field-effect devices -a useful combination”. Sensors and Actuators, 1990, pp.15-20.

[16] Kalman, E-L., Lofvendahl, A., Winquist, F. and Lundstrom, I. (2000), "Classification of complex gas mixtures from automotive leather using an electronic nose", AnalyticaChimicaActa, Vol. 403, pp. 31-8.

[17] SHIERS, V. P. Electronic nose technology - evaluations and developments for the food industry. In: MAARSSEN (Ed), Food Ingredients Europe: Conference Proceedings1995. Frankfurt: Miller Freeman Technical, pp. 198-200 (1995)

[18] E. H. Oh, H. S. Song and T. H. Park, "Recent Advances in Electronic and Bioelectronic Noses and Their Bio- medical
Applications," Enzyme and Microbial Technol-ogy, Vol. 48, No. 6, 2011, pp.427-437.

[19] Albert, K.J. and Lewis, N.S., "Cross reactive chemical sensor arrays", Chem. Rev., Vol. 100, 2000, pp. 2595-626.

[20] Pearce, T.C., Schiffman, S.S., Nagle, H.T. and Gardner, J.W., "Handbook of Machine Olfaction", Wiley-VCH, Weinheim, 2003.

[21] Nagle, H.T., Gutierrez-Osuna, R. and Schiffman, S.S., "The how and why of electronic hoses", IEEE Spectrum, Vol. 35 No. 9,1998, pp. 22-31.

[22] Schaller, E., Bosset, J.O. and Escher, F. (1998), "Electronic noses and their application to food", Lebensmittel- Wissenschaft undTechnologie, Vol. 31 No. 4, pp. 305-16.

[23] R. O. Gutierrez, "Pattern Analysis for Machine Olfaction: A Review," IEEE Sensors Journal, Vol. 2, No. 3, 2006, pp. 189-202

[24] D. L. G. González, R. Aparicio and G. Y. Aceites, "Sen- sors: From Biosensors to the Electronic Nose," Grasas y Aceites, Vol. 53, No. 1, 2002, pp. 96-114.

[25] B.Lakshmi, K.ParishVenkata Kumar, Dr.KNageswara Rao, ShahnazBanu, Simulation of Artificial Noses for the Automated Detection and Classification of Organic Compounds, International Journal of Computer and Information Technologies, Vol 4(2), 2013,233-237.

[26] T.Rajthilak,M.Sasikumar, "Artificial Neural Networks for E-Nose", Research Journal on Computer Engineering, 2008, pp.69-73.

[27] Miguel Peris, Laura Escuder-GilabertA 21st century technique for food control: Electronic noses

[28] L. Marilley,S. Ampuero,T. Zesiger, M.G.Casey, Int. Dairy J.14 (2004) 849

[29] W. Cynkar, D. Cozzolino, B. Dambergs, L. Janik, M. Gishen, Sens. Actuators B: Chem. 124 (2007) 167.

[30] A. Hernández-Gómez, J. Wang, G. Hu, A. García-Pereira, J. Food Eng. 85 (2008) 625

[31] M. Lebrun, A. Plotto, K. Goodner, M.N. Ducampa, E. Baldwin, Postharvest Biol. Technol. 48 (2008) 122.

[32] J. Brezmes, E. Llobet, X. Vilanova, J. Orts, G. Saiz, X. Correig, Sens. Actuators B: Chem. 80 (2001) 41.

[33] U. Herrmann, T. Jonischkeit, J. Bargon, U. Hahn, Q.Y. Li, C.A. Schalley, E. Vogel, F. Vögtle, Anal. Bioanal. Chem. 372 (2002) 611

[34] A.Z. Berna, J. Lammertyn, S. Saevels, C. Di Natale, B.M. Nicolaï, Sens. Actuators B: Chem. 97 (2004) 324.

[35] S. Saevels, J. Lammertyn, A.Z. Berna, E.A. Veraverbeke, C. Di Natale, B.M. Nicolai, Postharvest Biol. Technol. 31 (2004) 9.

[36] A. Hernández-Gómez, G. Hu, J.Wang, A. García-Pereira, Comput. Electron. Agr. 54 (2006) 44

[37] A. Hernández-Gómez, J.Wang, G. Hu, P. García-Pereira, LWTFood Sci. Technol. 40 (2007) 681

[38] S. Benedetti, S. Buratti, A. Spinardi, S. Manninoa, I. Mignani, Postharvest Biol. Technol. 47 (2008) 181.

[39] C. Di Natale, G. Olafsdottir, S. Einarsson, E. Martinelli, R. Paolesse, A. D'Amico, Sens. Actuators B: Chem. 77 (2001) 572

[40] Paul E. Keller, Lars J. Kangas, Lars H. Liden, SherifHashem, Richard T. Kouzes "Electroic Nose and their Application." IEEE Northcon/Technical Applications Conference (TAC'95) in Portland, OR, USA on 12 October 1995.

[41] K. Pope, "Technology Improves on the Nose As Science Tries to Imitate Smell," Wall Street Journal, pp. B1-2, 1 March 1995.

[42] P.E. Keller, R.T. Kouzes, L.J. Kangas, and S. Hashem, "Transmission of Olfactory Information for Telemedicine," Interactive Technology and the New Paradigm for Healthcare, R.M. Satava, K. Morgan, H.B. Sieburg, R. Mattheus, and J.P. Christensen (ed.s), IOS Press, Amsterdam, 1995, pp. 168-172.

[43] R.J. Lauf and B.S. Hoffheins, "Analysis of Liquid Fuels Using a Gas Sensor Array,” Fuel , vol. 70, pp. 935-940, 1991.

[44] M.A. Markom, A.Y. MdShakaff, A.H. Adom, M.N. Ahmad, WahyuHidayat , A.H. Abdullah, N. Ahmad Fikri "Intelligent electronic nose system for basal stem rot disease detection" Computers and Electronics in Agriculture 66 (2009) 140-146. 